\title{
Expressional Regulation of Angiopoietin-1 and -2 and the Tie-1 and -2 Receptor Tyrosine Kinases during Cutaneous Wound Healing: A Comparative Study of Normal and Impaired Repair
}

\author{
Heiko Kämpfer, Josef Pfeilschifter, and Stefan Frank \\ Zentrum der Pharmakologie, Klinikum der Johann Wolfgang Goethe-Universität, Frankfurt am Main, Germany
}

SUMMARY: It has become evident that a closely regulated presence of vascular endothelial growth factor (VEGF) and angiopoietin (Ang) factors determines the fate of blood vessel formation during angiogenesis. As angiogenesis is central to a normal wound-healing process, we investigated the regulation of Ang-1 and -2 and the related tyrosine kinase with immunoglobulin and epidermal growth factor homology (Tie)-1 and -2 receptors during normal repair in Balb/c mice and diabetes-impaired wound healing conditions in genetically diabetic $(d b / d b)$ mice. For both normal and impaired healing conditions, we observed a constitutive expression of Ang-1, which was paralleled by an increase of Ang-2 upon injury. Whereas the observed Ang-2 expression declines from Day 7 after injury in control mice, diabetic-impaired healing was characterized by still increasing amounts of Ang-2 at these time points. Furthermore, Tie-1 was strongly induced during repair with a prolonged expression in diabetic mice, whereas Tie-2 expression was constitutive during normal repair but completely absent in diabetes-impaired healing. The overexpression of Ang-2 in the presence of markedly reduced VEGF in wounds of diabetic mice was associated with a dramatic decrease in endothelial cell numbers compared with normal healing as assessed by analysis of the endothelium-specific markers CD31 and von Willebrand factor, whereas the lymphatic endothelium remained stable as determined by expression of VEGF receptor-3 (VEGFR-3/FIt-4). (Lab Invest 2001, 81:361-373).

$E$ arly vascular development (vasculogenesis) comprises complex processes such as regulated proliferation, differentiation, and migration of endothelial cells that finally coalesce to form a primitive vascular network (Risau, 1997). Subsequently, the immature, primitive network undergoes remodeling processes involving sprouting, branching, differential growth of vessels, and recruitment of supporting cells (angiogenesis) to form a mature cardiovascular system (Risau, 1997). Once differentiated, the mature vasculature remains relatively stable. Central for regulation of these processes are two families of growth factors, the vascular endothelial growth factor (VEGF) and the recently described angiopoietin (Ang) family. Five VEGF relatives have been described for mammals (VEGF A through D, and placenta growth factor) that exert biological functions via three related receptor tyrosine kinases (VEGFR-1/Flt-1, VEGFR-2/KDR/ Flk-1, VEGFR-3/Flt-4) (Gale and Yancopoulos, 1999). Generation of knock-out animals for VEGF and its receptors revealed a critical role for vasculogenesis during embryonic development (Carmeliet et al, 1996;

Received November 13, 2000

This work was supported by a grant of the Deutsche Forschungsgemeinschaft (SFB 553) and the Adolf Messer-Stiftung.

Address reprint requests to: Dr. Stefan Frank, Institut für Allgemeine Pharmakologie und Toxikologie, Klinikum der JWG-Universität Frankfurt/M., Theodor-Stern-Kai 7, D-60590 Frankfurt/M., Germany. E-mail:S.Frank@em.uni-frankfurt.de
Ferrara et al, 1996; Fong et al, 1995; Shalaby et al, 1995). Comparable to the VEGF family in the specificity for the vascular endothelium, the recently discovered angiopoietins (Ang-1 through -4) (Davis et al, 1996; Maisonpierre et al, 1997; Suri et al, 1996; Valenzuela et al, 1999) are essentially involved in maturation, stabilization, and remodeling of vessels. Angiopoietin action is targeted towards the vascular endothelium, because both related tyrosine kinase with immunoglobulin and epidermal growth factor homology (Tie)-1 and Tie-2 receptors are specifically expressed in vascular endothelial cells (Dumont et al, 1995; Maisonpierre et al, 1993). The four identified angiopoietins use the associated receptor tyrosine kinase Tie-2. Although a functional ligand for the related Tie-1 receptor remains unidentified, it is well known that Tie-1 functions in controlling vascular endothelial cell integrity. Hence, embryos deficient in Tie-1 die because of the resulting edema and localized hemorrhage (Puri et al, 1995; Sato et al, 1995). In vivo studies revealed a close interaction of the Ang-1/Tie-2 system with VEGF for vascular development. In Ang-1 or Tie-2-deficient embryos, a VEGF-mediated primitive vasculature develops, but embryos fail to remodel and stabilize the preformed vasculature, which finally leads to embryonic death (Dumont et al, 1994; Sato et al, 1995; Suri et al, 1996). Transgenic overexpression of Ang-1 in the skin of mice leads to more numerous dermal capillaries and venules that are highly branched and characterized by increased diameters. 
However, the vasculature displays structural integrity and functionality (Suri et al, 1998). In contrast to skin-targeted VEGF expression that causes hyperpermeability and leakiness of vessels, Ang-1 overexpression results in a nonleaky vasculature, even under induced inflammatory conditions (Thurston et al, 1999). In line with a potentially stabilizing role of Ang-1 for mature vessels, Ang-1 is found to be constitutively expressed in the adult (Maisonpierre et al, 1997). By contrast, Ang-2, representing a natural Tie-2 antagonist, is highly induced at sites of vascular remodeling in the adult, such as the female reproductive tract (Maisonpierre et al, 1997), or during tumor growth and metastasis (Holash et al, 1999), suggesting that Ang-2 mediates a destabilization of existing vessels leading to a more plastic state. This situation then converts to active vascular remodeling in the presence of VEGF or to regression of frank vessels in the absence of VEGF (Holash et al, 1999; Maisonpierre et al, 1997).

A closely controlled angiogenic situation is also involved in tissue repair processes. The closure of cutaneous wounds comprises complex tissue movements such as hemorrhage, re-epithelialization, granulation tissue formation, and angiogenesis (Martin, 1997). Angiogenesis is central to granulation tissue formation because the ingrowth of newly formed vessels is needed to ensure the supply of oxygen and nutrients to the regenerating tissue. It is well known that keratinocyte-derived VEGF expression is associated with a normal repair (Brown et al, 1992) or nearly absent in diabetes-impaired healing (Frank et al, 1995). However, the role of the angiopoietins and their related Tie receptors within the process of cutaneous repair largely remains unknown. Findings described in the current study demonstrate a constitutive expression of the Ang-1/Tie-2 system during tissue regeneration. The functionality of the Ang-1/Tie-2 interaction is most likely to be interrupted by a dramatic induction of Ang-2 upon skin injury. In line with the observation of a temporally close control of Ang-2 during normal healing, we found a prolonged expression of Ang-2 in diabetes-impaired wound repair. Moreover, elevated levels of Ang-2 were associated with clearly reduced expression of the Tie-2 receptor during impaired healing. Additionally, reduced VEGF levels observed in wounds of diabetic $(d b / d b)$ mice (Frank et al, 1995; this study) clearly correlate with a dramatically reduced formation of vascular endothelium in the presence of an elevated Ang-2 expression in these mice, which are characterized by severely disturbed woundhealing conditions.

\section{Results}

Dysregulated Expression of Ang-1, Ang-2, Tie-1, and Tie-2 during Impaired Wound Healing in Diabetic (db/db) Mice

In contrast to VEGF expressional regulation (Brown et al, 1992; Frank et al, 1995), regulation of the angiopoietin/Tie ligand-receptor system in tissue regeneration during cutaneous wound repair remains unknown.
Therefore, we investigated the expression pattern of Ang- 1 and -2 and their related receptors Tie- 1 and -2 during normal and impaired repair. Genetically diabetic mice $(d b / d b)$ that develop diabetes in a manner similar to the human adult onset of diabetes (Coleman, 1982) were used as a model of severely disturbed wound-healing conditions. Wounds were isolated by excision of the individual wounded area, including the epithelial wound margins. Thus, the co-isolation of unaffected skin regions, which might confound the interpretation of data obtained from wound tissue analysis, was reduced to a minimum. As shown in Figure 1A, Ang-1 was found to be constitutively expressed during normal repair at the mRNA and also at
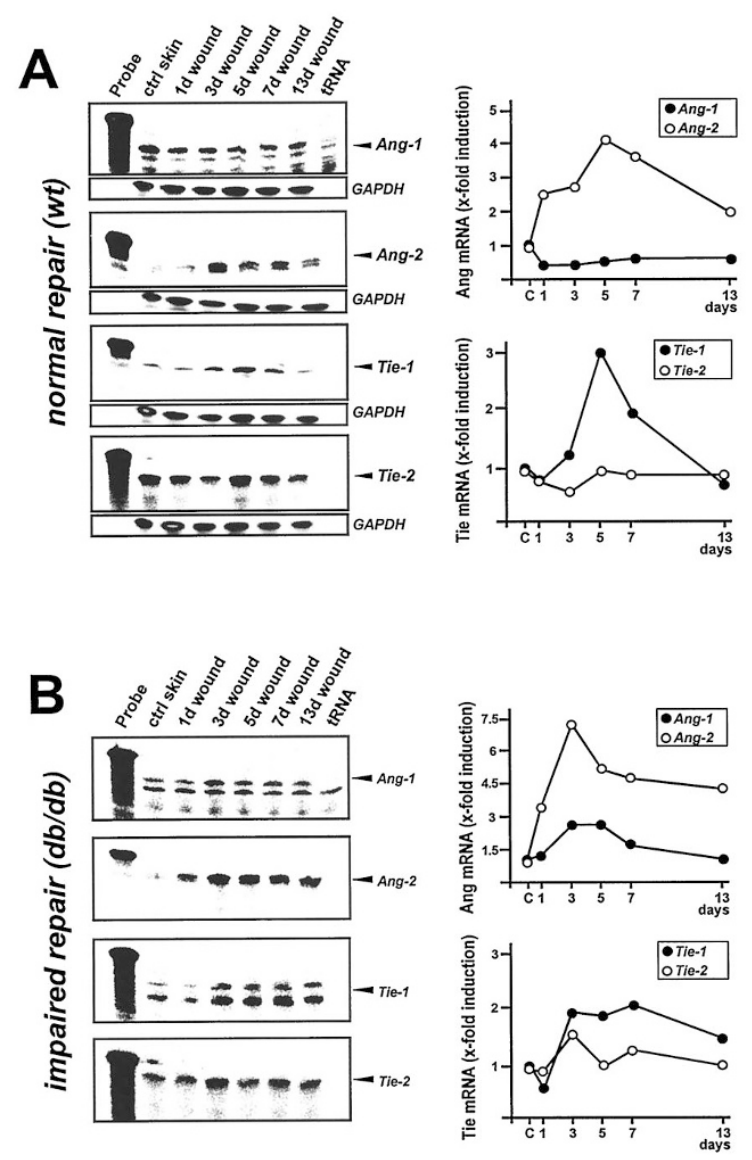

Figure 1.

Regulation of Ang-1, Ang-2, Tie-1, and Tie-2 mRNA expression during normal and diabetes-impaired wound repair. Expression of angiopoietins (Ang-1 and -2) and related receptors (Tie-1 and -2) during wound healing is shown for Balb/c control mice (A, normal repair, $w t)$ and genetically diabetic $d b / d b$ mice $(B$, impaired repair, $d b / d b)$ as indicated. Total cellular RNA $(20 \mu \mathrm{g})$ from nonwounded and wounded back skin of control mice (wt), or $\mathrm{db} / \mathrm{db}$ mice was analyzed by RNase protection assay at the indicated time points after injury for the presence of Ang-1, Ang-2, Tie-1, or Tie-2 mRNA (left panels). Sixteen wounds $(n=16)$ from the backs of four animals were excised for each experimental time point and used for RNA isolation. ctrl skin refers to nonwounded skin of $B a l b / c$ and $d b / d b$ mice. One representative experiment is shown; $1000 \mathrm{cpm}$ of the hybridization probe were used as a size marker (probe). Expression of glyceraldehyde-3-phosphate dehydrogenase (GAPDH) mRNA is shown as a loading control. The degree of regulation as assessed by Phospholmager analysis of the radiolabeled gels is shown schematically in the right panels. For the right panels, note that every experimental time point represents a total of 32 wounds $(n=32)$ from two independent animal experiments. 
the protein level (Fig. 2A). Accordingly, we observed an unaltered presence of the Ang-1 receptor tyrosine kinase Tie-2 in uninvolved control skin and during tissue regeneration in Balb/c mice (wt) (Fig. $1 \mathrm{~A}$ and Fig. 2D). Not unexpectedly, Ang-2 was largely induced (4-fold) upon injury in control animals (wt) (Fig. 1A and Fig. 2B). Additionally, we observed the Tie-1 receptor to be up-regulated (3-fold at the mRNA level) during granulation tissue formation between Day 3 and 7 after injury in Balb/c animals (wt) (Fig. 1A and Fig. 2C). Determination of Ang/Tie expression patterns during diabetes-impaired healing revealed a dysregulation compared with wounds isolated from control animals. Predominantly, we found increased Ang-1 and Ang-2 expression at the mRNA levels in diabetic wounds (Fig. 1B) compared with normal wounds (Fig. 1A), whereas Tie-1 and Tie-2 mRNA expression remained comparable during normal (Fig. 1A) and impaired repair (Fig. 1B). However, a more severe diabetesassociated dysregulation takes place at the protein level. Notably, mRNA induction of Ang-1 was not followed by elevated protein levels (Fig. 2A). Whereas the Ang-1-associated receptor Tie-2 was strongly expressed in normal skin (wt) (Fig. 2D, upper panel), Tie-2 was only weakly present in uninvolved skin of $d b / d b$ mice (Fig. 2D, lower panel). Moreover, Tie-2specific signals completely disappeared upon injury in these animals (Fig. 2D, lower panel).

Consistent with increased mRNA levels, we observed elevated protein expression for Ang-2-specific proteins at the later stages of repair (Days 7 and 13, respectively) associated with impaired healing (Fig. $2 \mathrm{~B}$, third panel). Finally, we investigated Ang-2 and Tie-1 protein levels at Day 10 after wounding because the granulation tissue is characterized by formation of new blood vessels during this phase of repair. To this end, four wounds each $(n=4)$ were isolated from the backs of three individual animals at Day 10 after wounding. For each individual animal, the wounds ( $n$ $=4$ ) were pooled and analyzed for expression of
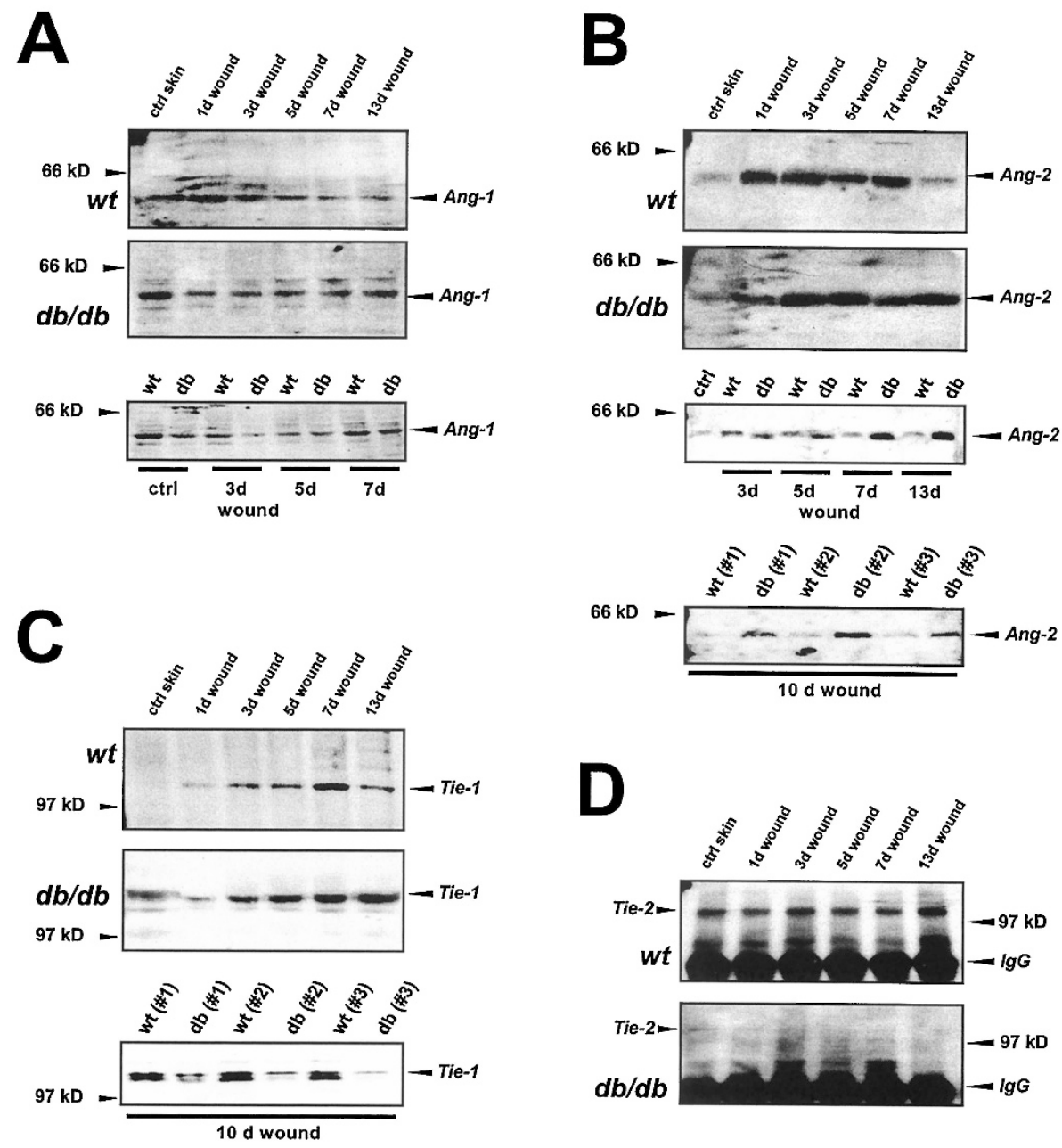

Figure 2.

Dysregulation of Ang-1, Ang-2, Tie-1, and Tie-2 protein expression during wound healing in $d b / d b$ mice. Total protein $(50 \mu \mathrm{g})$ from lysates of nonwounded and wounded back skin (Days 1, 3, 5, 7, 10, and 13 after injury, indicated at the top of each lane) isolated from Balb/c mice (wt) and diabetic mice ( $d \mathrm{db} / \mathrm{db}$ ) as indicated were analyzed by immunoblotting for the presence of Ang-1 (A), Ang-2 (B), Tie-1 (C), and Tie-2 (D) specific protein. Note that Tie-2 (D) represents an immunoprecipitation experiment. Eight wounds $(n=8)$ from the backs of four animals were excised for each experimental time point and used for protein isolation. For the individual animals shown in B and C (lower panels), four wounds each $(n=4)$ were pooled for analysis. ctrl skin refers to nonwounded skin of Balb/c and $d b / d b$ mice. Ang-1, Ang-2, Tie-1, or Tie-2 protein is indicated by an arrow. 
Ang-2 and its receptor Tie-1 (Fig. 2, B and C, lower panels, wt \#1-3, db \#1-3). We could confirm elevated levels of Ang-2 protein in wounds from diabetic animals compared with normal healing conditions (Fig. 2B, lower panel). By contrast, although the expression kinetics for Tie-1 protein as assessed by Western blot analysis suggested elevated amounts of Tie-1 protein in wounds from diabetic mice (Fig. 2C, middle panel), it is important to note that a direct comparison of wound-derived Tie-1 expression in normal versus diabetic animals revealed markedly reduced amounts of Tie-1 protein at later phases of impaired skin repair (Fig. 2C, lower panel). Taken together, we found reduced Tie-1 and -2 protein expression during impaired repair, which was paralleled by an increased expression of the ligand Ang-2 during later phases of the healing process.

\section{Relative Ang-2/-1 and Tie-1/-2 Expression Levels in Wounds from Normal and Diabetic Mice}

At this point of research, the relative ratios of Ang-1/ Ang-2 and, moreover, Tie-1/Tie-2 mRNA expression remained unclear. Especially, the nonlinear detection of Ang and Tie proteins using the enhanced chemiluminescence (ECL) system did not allow the analysis of subtle expressional regulations. Moreover, because the baseline expression levels of Ang-1 and -2 and, additionally, their Tie-1 and-2 receptors were different (Fig. 1), and the relative ratio of Ang-2 versus Ang-1 is suggested to be critical in regulating Ang function, we determined relative Ang and Tie mRNA levels at the wound site. To this end, we analyzed total wound RNA $(20 \mu \mathrm{g})$ by quantitative RNase protection assay for relative Ang-1/Ang-2 or Tie-1/Tie-2 mRNA expression levels (see "Materials and Methods" section). For Table 1, it is important to note that every data point represents a total of 32 wounds $(n=32)$ isolated from eight different animals of two independent experimental series. We observed two striking differences in Ang-1/Ang-2 regulation in normal compared with diabetic animals. First, and shown in Table 1A, normal healing was characterized by a moderate excess of Ang-2 compared with Ang-1 mRNA (3- to 4-fold between Days 1and 7), whereas diabetic repair revealed a much stronger increase in Ang-2/Ang-1 mRNA relative ratios (10- to 12-fold between Days 1 and 7) compared with normal healing. Moreover, increased Ang-2/Ang-1 ratios persisted until Day 13 after wounding. Thus, these data suggest a dysregulation of Ang-1/Ang-2 expression levels during impaired repair. In addition, Ang-1/Ang-2 relative ratios were disturbed in nonwounded skin of diabetic animals compared with healthy mice. Because skin of normal mice was characterized by higher Ang-1 mRNA levels (Ang-2/Ang-1 relative ratio: 0.71), diabetic animals showed markedly elevated amounts of Ang-2 mRNA in nonwounded skin per se (Ang-2/ Ang-1 relative ratio: 3.12 ). Finally, analysis of relative Tie-1/-2 expression levels revealed a more pronounced increase of Tie-1 mRNA amounts in relation to Tie-2 mRNA levels in normal wound healing (Table 1).

\section{Ang-2 mRNA Is Strongly Expressed in the Panniculus Carnosus}

As a next step, we investigated the localization of Ang-2 mRNA at the wound site. As Ang-2 protein is well established to be secreted, we used the in situ hybridization (ISH) technique to determine potential cellular sources of Ang-2 mRNA expression. For this purpose, we isolated wounds from normal and diabetic mice at Day 5 after injury because Ang-2 expres-

Table 1. Relative Ratios of Ang-2/-1 and Tie-1/-2 mRNA Levels during Normal and Impaired Repair ${ }^{\mathrm{a}}$

\begin{tabular}{|c|c|c|c|c|c|c|}
\hline \multirow[b]{2}{*}{$A$} & \multirow[b]{2}{*}{ Ctrl } & \multicolumn{5}{|c|}{ Day Post-Wounding (Wild-Type) } \\
\hline & & 1 & 3 & 5 & 7 & 13 \\
\hline Ang-1 & 1653 & 781 & 656 & 892 & 996 & 684 \\
\hline Ang-2 & 1171 & 2300 & 2765 & 3586 & 2801 & 2109 \\
\hline Ang-2/-1 relative ratio & 0.71 & 2.95 & 4.21 & 4.02 & 2.81 & 3.08 \\
\hline Tie-1 & 3156 & 2211 & 3410 & 6467 & 4082 & 2191 \\
\hline Tie-2 & 2786 & 1816 & 1498 & 2217 & 1792 & 1500 \\
\hline \multirow[t]{2}{*}{ Tie-1/-2 relative ratio } & 1.13 & 1.22 & 2.28 & 2.91 & 2.27 & 1.46 \\
\hline & & \multicolumn{5}{|c|}{ Day Post-Wounding (Diabetic) } \\
\hline B & Ctrl & 1 & 3 & 5 & 7 & 13 \\
\hline Ang-1 & 185 & 184 & 275 & 215 & 370 & 284 \\
\hline Ang-2 & 579 & 1848 & 3269 & 2164 & 2207 & 2166 \\
\hline Ang-2/-1 relative ratio & 3.12 & 10.00 & 11.89 & 10.07 & 5.97 & 7.63 \\
\hline Tie-1 & 526 & 528 & 687 & 537 & 867 & 880 \\
\hline Tie-2 & 384 & 437 & 543 & 395 & 351 & 524 \\
\hline Tie-1/-2 relative ratio & 1.37 & 1.21 & 1.27 & 1.36 & 2.47 & 1.68 \\
\hline
\end{tabular}

Ang, angiopoietin; Tie, tyrosine kinase with immunoglobulin and epidermal growth factor homology domains; Ctrl, control; PSL, signal intensity per area.

a Data are presented as absolute Phospholmager PSL counts, which directly correlate to the amount of the analyzed mRNA species. 
sion appeared to be strongly elevated at this phase of repair. As shown in Figure $3, A$ and $C$ (left panels), we observed a strong expression of Ang-2 mRNA in smooth muscle cells of the panniculus carnosus 5 days after injury. Because the initial excision of skin tissue also included the panniculus carnosus (see "Materials and Methods" section), Ang-2 mRNAexpressing cells were primarily located at the wound margins but also within the regenerating smooth muscle layer directly underlying the developing granulation tissue. Please note that the right panels ( $D$ and $F$ ) represent directly neighbored sections to panels $A$ and $\mathrm{C}$, which are hybridized by a nonsense ("scrambled") oligonucleotide followed by immunodetection to serve as a negative control. Additionally, we used 10-day wound tissues from normal mice as negative controls for Ang-2 mRNA expression (data not shown). Furthermore, the observed expression of Ang-2 in smooth muscle cells of the regenerating panniculus carnosus provided an explanation for the strong signals of Ang-2-specific protein which could be detected by Western blot analysis (Fig. 2B).

\section{Impaired Repair Is Clearly Associated with a Dramatic Decrease in Formation of Vascular Endothelium}

As a next step, we investigated a possible functional consequence of the observed dysregulated expression patterns of the Ang/Tie ligand-receptor system for vascular remodeling and, subsequently, the angiogenic response during disturbed tissue repair. Thus, we determined expression of CD31, which represents a highly specific marker for vascular and lymphatic endothelial cells. As shown in Figure 4, we assessed a markedly decreased expression of CD31 mRNA during diabetes-impaired wound repair $(d b / d b)$ (Fig. 4, A and $\mathrm{B})$, which was paralleled by a dramatic reduction in CD31 protein synthesis (Fig. 4, C and D). During normal repair $(w t)$, CD31 mRNA and protein expression was induced early after injury (4- to 5-fold), thus reflecting robust angiogenic processes at the wound site. By contrast, induction of CD31 mRNA expression was only transient in diabetic animals $(d b / d b)$ with a markedly delayed and, moreover, dramatically reduced expression of CD31 (Fig. 4).

As shown in Figure 5 (left panel), we confirmed the vascular endothelium as a cellular target for Ang actions by immunohistochemistry because we could detect expression of the Tie-2 receptor to be restricted to endothelial cells lining developing blood vessels located within the regenerating panniculus carnosus. To further strengthen our biochemical data (obtained by immunoblot analysis of CD31-specific proteins in normal versus diabetic wound-healing conditions), we determined CD31 expression in serial sections of wounds from normal (Fig. 5, middle panel) and diabetic (Fig. 5, right panel) mice at Day 10 after injury. Not unexpectedly, we could observe CD31positive vascular structures within the granulation tissue of normal animals (Fig. 5, middle panel). Please note that some of these vessels had collapsed during tissue embedding. By contrast, wounds of diabetic mice were characterized by a number of CD31stained cells that failed to organize into vessel-like structures (Fig. 5, right panel).

\section{Reduced Expression of VEGF Protein in Wounds of Diabetic (db/db) Mice}

Because we observed largely increased levels of Ang-2 and, subsequently, a nearly complete absence of angiogenic processes as assessed by CD31 expression at Days 5,7 , and 13 of repair in $d b / d b$ mice compared with Balb/c animals (wt), we hypothesized that this observation might be in line with a model of angiogenic remodeling proposed by Maisonpierre and colleagues (1997), namely that interruption of Ang-1/ Tie-2 functional interaction most likely results in blood vessel regression in the absence of VEGF. Because we have determined VEGF mRNA expression to be nearly absent in wounds of $d b / d b$ mice after injury (Frank et al, 1995), we now investigated VEGF protein levels in total wound lysates from wounds of control $(w t)$ and diabetic mice $(d b / d b)$ using a sensitive ELISA method (R\&D Systems, Wiesbaden, Germany). Indeed, we could measure markedly decreased VEGF protein levels (maximal levels, approximately 120 pg VEGF $_{165} / 50 \mu \mathrm{g}$ wound lysate protein) during impaired repair in $d b / d b$ mice compared with control animals (maximal levels, approximately 350 pg VEGF ${ }_{165} / 50 \mu \mathrm{g}$ wound lysate protein) as shown in Figure 6.

\section{Expression of the Lymphatic Endothelium-Specific VEGFR-3/FIt-4 Is Not Altered during Impaired Repair}

Finally, we wanted to exclude that the overall and severely impaired wound-healing conditions observed for $d b / d b$ mice might underlie or mimic the observed correlation between disrupted angiogenic processes and dysregulation of the Ang/Tie system in the absence of VEGF. This was important to us because $d b / d b$ mice suffer from a series of physiological disturbances such as hyperinsulinemia, obesity, impaired reproduction, decreased thermogenesis, reduced growth, and high levels of circulating corticosterone (Leibel et al, 1997). Therefore, we separated the complete endothelium-derived response as measured by the pan-endothelial marker CD31 (Fig. 4) by determination of von Willebrand factor (vWF), which is predominantly expressed in vascular endothelium, and by VEGFR-3/FIt-4, which represents a specific marker of the lymphatic endothelium (Kukk et al, 1996; Lymboussaki et al, 1998). Thus, we hypothesized that an undisturbed VEGFR-3/FIt-4 expression (lymphatic response) in the presence of reduced expression of vWF (vascular response) would further strengthen a direct correlation between overexpression of Ang-2, the absence of VEGF, and, subsequently, loss of an angiogenic response in these animals. Confirming the results obtained for CD31 expression, we observed only a transient and weak expression of VWF response in diabetic animals $(d b / d b)$ upon wounding (about 2-fold, Days 1 to 3 after wounding), whereas normal wound repair (wt) was characterized by largely elevated levels 

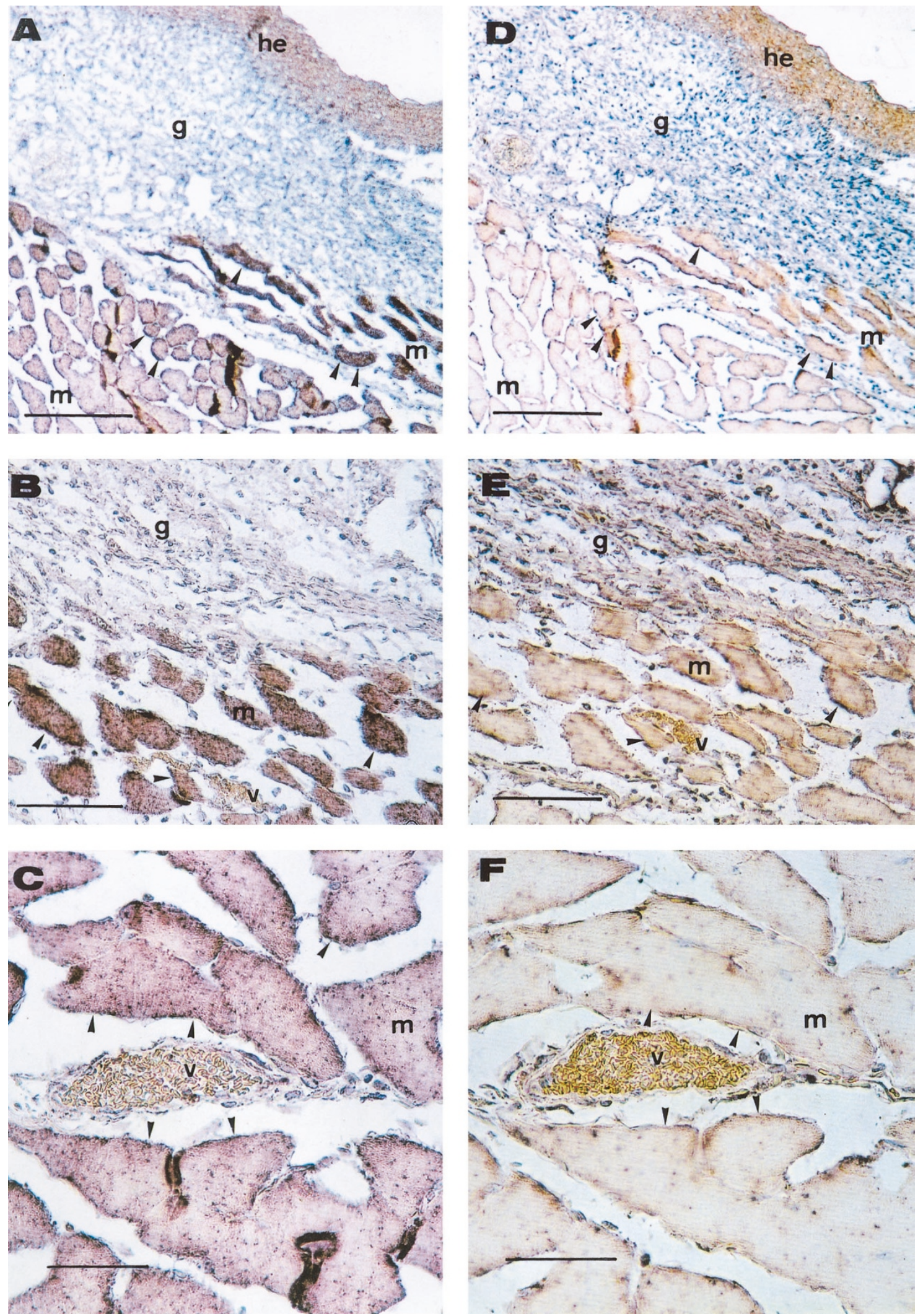

Figure 3.

Localization of Ang-2 mRNA expression at the wound site. Paraformaldehyde (PFA)-fixed serial sections from 5-day mouse wounds isolated from Balb/c control animals were analyzed for the presence of Ang-2 mRNA expression by in situ hybridization. Sections were incubated with an FITC-labeled antisense oligonucleotide specific for Ang-2 mRNA ( $A$ to C). Note that directly neighboured sections ( $D$ to F) were incubated with a nonsense oligonucleotide as a negative control. All sections were stained with an alkaline phosphatase-coupled anti-FITC antibody using NBT/formazan as a chromogenic substrate. Nuclei were counterstained with hematoxylin Scale bars are $100 \mu \mathrm{m}$ for $\mathrm{A}$ and D, $50 \mu \mathrm{m}$ for B and E, and $25 \mu \mathrm{m}$ for C and F. Strongly immunopositive signals (A to C) and the corresponding negative controls (D to F) are indicated with arrows. g, granulation tissue; he, hyperproliferative epithelium; m, muscle; v, vessel. 

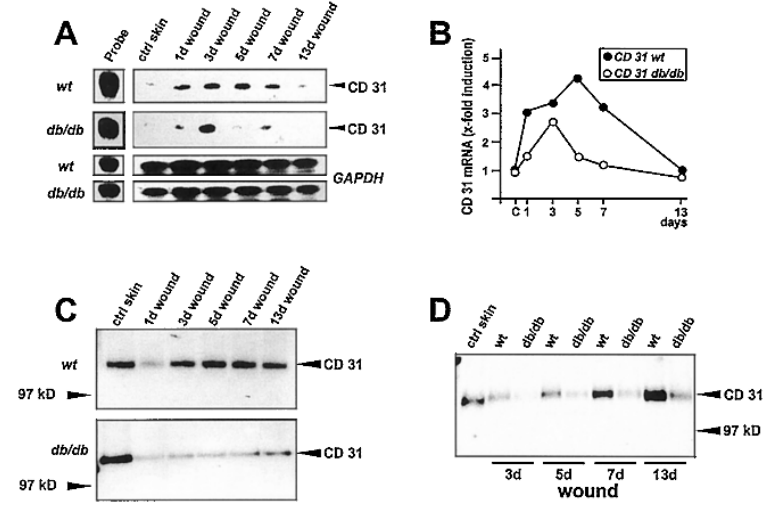

Figure 4.

Severely delayed endothelial response during diabetes-impaired wound healing. A, Expression of the endothelial marker CD31 during wound healing is shown for Balb/c mice (wt) and diabetic mice $(d b / d b)$ as indicated. Total cellular RNA $(20 \mu \mathrm{g})$ from nonwounded and wounded back skin of Balb/c mice (wt) or diabetic mice $(d b / d b)$ was analyzed by RNase protection assay at the indicated time points after injury for the presence of CD31 mRNA (left panels). Sixteen wounds $(n=16)$ from the backs of four animals were excised for each experimental time point and used for RNA isolation. ctrl skin refers to nonwounded skin of control and $d b / d b$ mice. One representative experiment is shown; $1000 \mathrm{cpm}$ of the hybridization probe were used as a size marker (probe). Expression of GAPDH mRNA is shown as a loading control. The degree of regulation as assessed by Phospholmager analysis of the radiolabeled gels is shown schematically in B. For B, note that every experimental time point represents a total of 32 wounds $(n=32)$ from two independent animal experiments. C and D, Total protein $(50 \mu \mathrm{g})$ from lysates of nonwounded and wounded back skin (Days 1, 3, 5, 7, and 13 after injury, as indicated) isolated from Balb/c mice (wt) and diabetic mice $(d b / d b)$ as indicated were analyzed by immunoblotting for the presence of CD31 specific protein. Eight wounds $(n=8)$ from the backs of four animals were excised for each experimental time point and used for protein isolation. ctrl skin refers to nonwounded skin. CD31 protein is indicated by an arrow.

of vWF mRNA expression (4 to 6-fold, until Day 13 after wounding) (Fig. 7A). By contrast, VEGFR-3/FIt-4 expression, reflecting lymphatic endothelium, appeared to be similar in diabetic $(d b / d b)$ and normal $(w t)$ wounds (Fig. 7B), suggesting that, indeed, a dysregulated Ang/Tie/VEGF system specifically imbalances vascular remodeling processes.

\section{Discussion}

To overcome tissue damage, the process of cutaneous wound repair represents a highly ordered process that is characterized by overlapping phases of important tissue movements such as hemorrhage, inflammation, re-epithelialization, granulation tissue formation, and the late remodeling phase of repair. The early and acute phases of repair involve macrophage accumulation, fibroblast ingrowth, matrix deposition, and angiogenesis (Clark, 1996; Martin, 1997). These processes are driven in part by a complex mixture of growth factors and cytokines that are released coordinately into the area of injury. A strong angiogenic response has to be induced during cutaneous tissue regeneration because the migrating and proliferative fibroblasts within the developing granulation tissue must be supplied with oxygen and nutrients. Accordingly, wound healing is characterized by a strong induction of VEGF expression early after injury, which continues during the phase when granulation tissue normally occurs (Brown et al, 1992; Frank et al, 1995). VEGF action is mediated in a paracrine manner because keratinocytes of the epithelial margins and, to a smaller extent, also macrophages secrete VEGF into the wound site (Brown et al, 1992; Frank et al, 1995; Xiong et al, 1998). VEGF action is specifically targeted to the vascular endothelium by a restricted expression of the VEGFR-1 and -2 (Fong et al, 1995; Shalaby et al, 1995), whereas VEGF-C acts on VEGFR-3/FIt-4 expressed in lymphatic endothelial cells (Kukk et al, 1996; Lymboussaki et al, 1998). Because diabetes represents a major risk for cardiovascular diseases (Aronson and Rayfield, 1996), and, moreover, because collateral vessel development is well known to be impaired in diabetic patients especially in lower extremity ischemia (Towne, 1989), novel strategies such as adenoviral-mediated VEGF expression were used to restore neovascularization in diabetic animal experimental models (Rivard et al, 1999) and also in humans suffering from ischemic leg ulcers (Baumgartner et al, 1998). Because of the expression of recombinant VEGF, ischemic ulcers healed or markedly improved. However, as VEGF action on the endothelium represents not the whole story of angiogenesis, expression of VEGF caused lower-extremity edema consistent with VEGF-mediated increase in vascular permeability (Baumgartner et al, 1998).

Accordingly, recent findings indicate that the regulatory potencies mediating endothelial movements are not restricted to members of the VEGF family. A second family of endothelial cell-specific growth factors, termed "angiopoietins," has been identified (Davis et al, 1996; Maisonpierre et al, 1997; Suri et al, 1996; Valenzuela et al, 1999). Targeted genedisruption experiments revealed a crucial role for Ang-1 and Ang-2 and their related Tie-1 and -2 receptors for embryonic angiogenesis that follows the preceding vasculogenic processes. Thus, early vasculogenesis during embryonic development is mainly dependent on the presence of a closely regulated expression of VEGF-A and its receptors VEGFR-1, -2 , and -3 (Carmeliet et al, 1996; Dumont et al, 1998; Ferrara et al, 1996; Fong et al, 1995; Shalaby et al, 1995). Interestingly, murine embryos deficient for Ang- 1 or the Tie-1 and -2 receptors die as a result of defects that are associated with vessel maturation and stabilization processes, such as defective vessel remodeling, organization, and sprouting or poor vessel integrity leading edema and hemorrhage (Davis et al, 1996; Dumont et al, 1994; Puri et al, 1995; Sato et al, 1995; Suri et al, 1996). These in vivo studies indicate that the angiopoietin system acts in a complementary and coordinated manner with VEGF at later stages of vascular development. Accordingly, because wound healing represents one of the rare events where angiogenesis takes place in the adult, it was reasonable to assume a participation of Ang-1 and -2 for tissue regeneration. A recent study indicated the involvement of the Tie-2 receptor in repair of rat and mouse skin wounds because Tie-2 expression is reported to be induced during healing. Moreover, the detected 


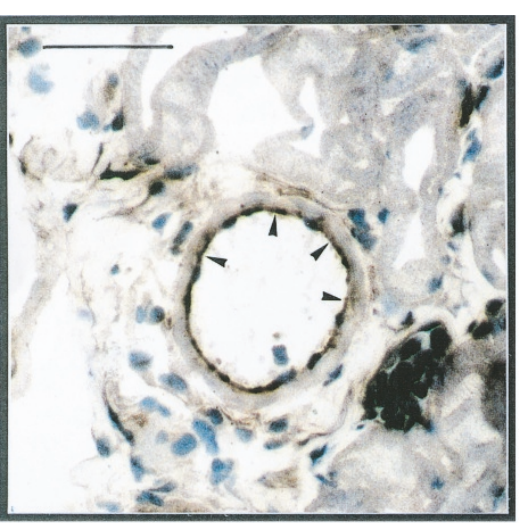

Tie-2 wild-type

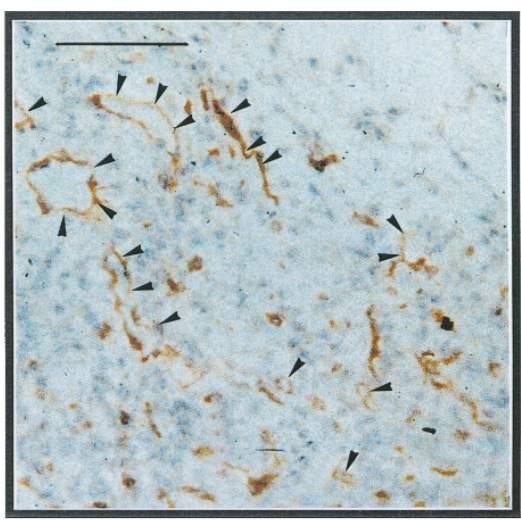

CD 31 wild-type

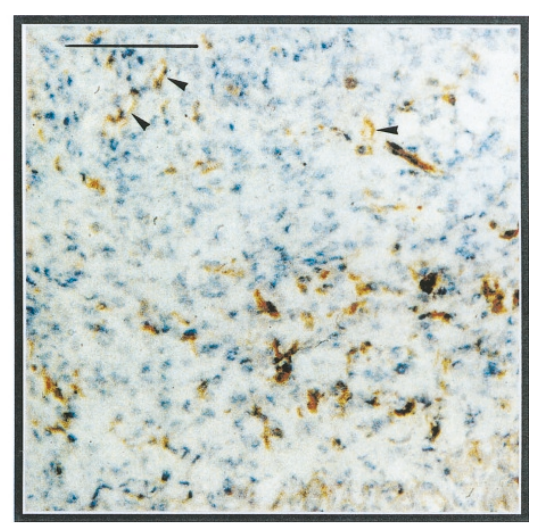

CD 31 diabetic

Figure 5.

Diabetes-impaired repair was characterized by reduced formation of CD31-positive vascular structures. Frozen sections ( $6 \mu \mathrm{m})$ from 5-day (Tie-2, wild-type) or 10-day (CD31, wildtype and diabetic) mouse wounds were incubated with polyclonal antisera directed against Tie-2 or CD31 as indicated. All sections were stained with the avidin-biotin-peroxidase complex system using 3-amino-9-ethylcarbazole as a chromogenic substrate. Nuclei were counterstained with hematoxylin. Scale bars are $25 \mu \mathrm{m}$ for (Tie-2, wild-type), and $75 \mu \mathrm{m}$ for (CD31, wild-type and diabetic). Strongly immunopositive signals are indicated with arrows.

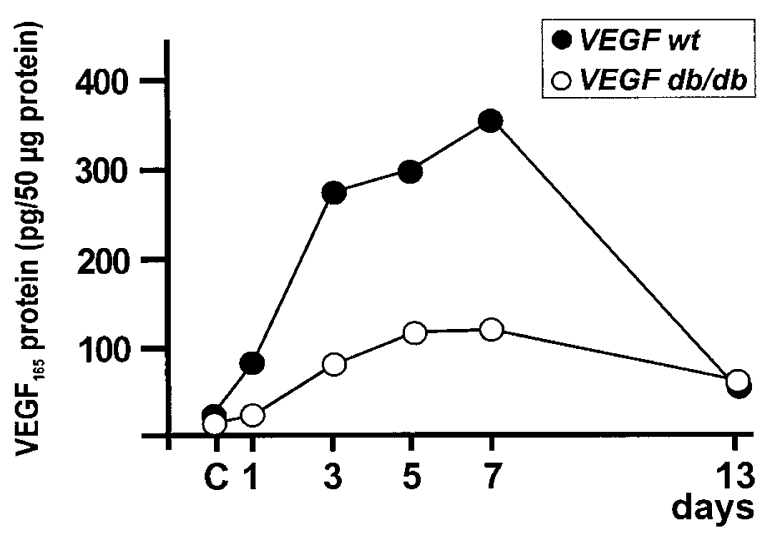

Figure 6.

Regulation of $\mathrm{VEGF}_{165}$ protein expression during normal and impaired healing. Total protein $(50 \mu \mathrm{g})$ from lysates of nonwounded and wounded back skin (Days 1, 3, 5, 7, and 13 after injury, as indicated) isolated from Balb/c mice $(w t)$ and diabetic mice $(d b / d b)$ were assessed for the total amount of $\mathrm{VEGF}_{165}$-specific proteins during wound repair as determined by ELISA. Eight wounds $(n=8)$ from the backs of four animals were excised for each experimental time point and used for protein isolation.

Tie-2 protein was tyrosine-phosphorylated, suggesting an active down-stream signaling (Wong et al, 1997). By contrast, data presented in this study confirm the overall presence of Tie-2 during repair, but we could not detect an induction of Tie-2 expression at the mRNA, or, for that matter, at the protein level. Notably, we found a large increase of Ang-2 upon injury in wounds of both control and diabetic animals. Because the more recently cloned Ang-2, which has been characterized as a natural occurring antagonist of Tie-2, does not mediate, but rather inhibits, Tie-2 tyrosine-phosphorylation (Maisonpierre et al, 1997), and, moreover, because inhibition of Ang-1/Tie-2 interactions represents a crucial prerequisite for a robust angiogenic response, the reported activated state of Tie-2 (Wong et al, 1997) in the presence of
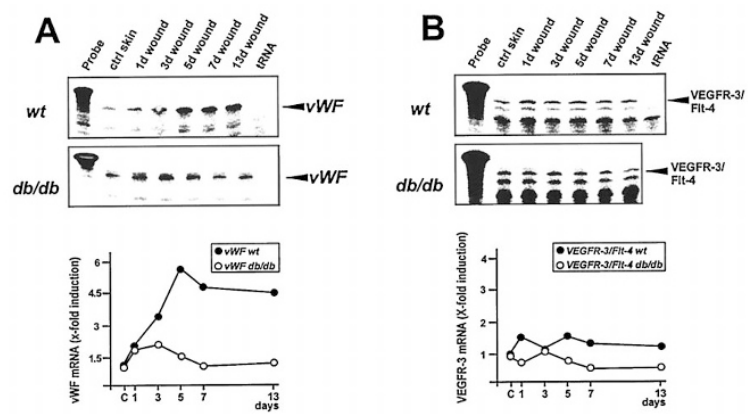

Figure 7.

Expression of lymphatic endothelium-derived VEGFR-3/FIt-4 mRNA during repair. Expression of von Willebrand factor (VWF) (A) and VEGFR-3/FIt-4 (B) during wound healing is shown for Balb/c control mice (wt) and diabetic mice $(d b / d b)$ as indicated. Total cellular RNA $(20 \mu \mathrm{g})$ from nonwounded and wounded back skin of Balb/c mice or $d b / d b$ mice was analyzed by RNase protection assay at the indicated time points after injury for the presence of VWF or VEGFR-3/FIt-4 mRNA (upper panels). Sixteen wounds $(n=16)$ from the backs of four animals were excised for each experimental time point and used for RNA isolation. ctrl skin refers to nonwounded skin of $B a l b / c$ and $d b / d b$ mice. One representative experiment is shown; $1000 \mathrm{cpm}$ of the hybridization probe were used as a size marker (probe). The degree of regulation as assessed by Phospholmager analysis of the radiolabeled gels is shown schematically in the lower panels. For the lower panels, note that every experimental time point represents a total of 32 wounds $(n=32)$ from two independent animal experiments.

Ang-2 during repair (this study) is in contrast to the proposed ability of Ang-2 to inactivate or block Tie-2dependent signaling (Maisonpierre et al, 1997).

Maisonpierre and colleagues (1997) proposed a model for the coordinated interaction between the VEGF and angiopoietin families mediating endothelial movements. Transgenic mice overexpression of Ang- 1 in the skin revealed its functions in regulating vessel stability (Suri et al, 1998). Although increased in branching numbers and diameter, vessels were characterized by normal cell-cell contacts between endothelial cells, pericytes, and fibroblasts, finally leading 
to a nonleaky and largely intact vasculature that was even leakage-resistant under induced inflammatory conditions (Thurston et al, 1999). Thus, Ang-1/Tie-2 are proposed to exert a stabilizing role for mature vessels, which is consistent with the observed constitutive expression of Ang-1 in the adult (Maisonpierre et al, 1997). Ang-2, the natural antagonist for Tie-2, is highly expressed at sites of vascular remodeling such as the female reproductive tract (Maisonpierre et al, 1997). Blockage of Ang-1/Tie-2 actions by Ang-2 leads to different vascular responses depending on the presence of VEGF. When VEGF is expressed in the presence of Ang-2, vessels respond with angiogenic actions like sprouting and ingrowth, but the absence of VEGF at Ang-2-destabilized vessels is found to induce vessel regression. This hypothesis is strongly supported by recent findings observed for tumor growth and metastasis, which also involve angiogenic processes in the adult (Holash et al, 1999; Stratmann et al, 1998).

Notably, regulation of Ang-1 and -2 during cutaneous wound healing (this study), which is, to our knowledge, the first study dealing with the potential regulation of these factors in tissue regeneration, clearly supports the above-mentioned hypothesis for regulation of angiogenesis. Consistent with previous data (Maisonpierre et al, 1997), we observed a constitutive expression of the Ang-1/Tie-2 system in uninvolved skin of control animals, indicating a role in blood vessel homeostasis. Interestingly, we observed a dysregulated balance for Ang-1/Ang-2 mRNA expression in nonwounded skin of diabetic mice. In these animals, Ang-2 mRNA levels were found to markedly exceed Ang-1 mRNA levels in the skin, whereas skin of healthy animals was characterized by a predominant Ang-1 mRNA expression. These data support the notion that, in the skin of diabetic mice, Ang-2 might interfere with the vessel-stabilizing properties of Ang-1. Moreover, the reduced Tie-2 levels in nonwounded skin of diabetic animals further suggest a possible disturbance of skin vessel stability in these animals.

Upon injury, we determined there to be a large induction of Ang-2 in the presence of a simultaneous increase in VEGF expression (Brown et al, 1992; Frank et al, 1995; this study). This coregulated increase clearly suggests an Ang-2-mediated destabilization of persisting blood vessels at the wound margins, which results in a robust angiogenic response during repair as high levels of VEGF protein are present at the wound site. In line with this observation, we subsequently detected a massive increase in endotheliumspecific CD31 expression during repair and, additionally, the formation of CD31-positive vascular structures within the granulation tissue. Notably, wound healing in genetically diabetic $d b / d b$ mice, which suffer from severe wound-healing disorders, was characterized by a strong and sustained induction of Ang-2 that even further increased during late healing. Consistent with the disturbed Ang-2 expressional kinetics observed for impaired healing, it was not unexpected that the absolute amounts of Ang-2 tran- scripts compared with Ang-1 mRNA levels were markedly increased in wounds of diabetic compared with healthy animals. Thus, the observed injury-mediated reduction of Tie-2 expression in diabetic wounds in combination with high Ang-2 levels in the absence of a strong VEGF induction suggested a destabilization of blood vessels that finally leads to vessel regression. Indeed, an angiogenic response was nearly absent in $d b / d b$ mice during repair because CD31 expression and the formation of CD31-positive vascular structures was markedly reduced compared with normal healing. In addition, expression of both Tie receptors is found to be restricted to the vascular endothelium (Dumont et al, 1995; Maisonpierre et al, 1993). Accordingly, we observed strongly reduced amounts of Tie-1 and Tie-2 protein in wound tissues from diabetic mice compared with healthy animals. Thus, the reduced expression of Tie-1 and -2 during impaired healing represents a second line of evidence that supports the notion of severely impaired angiogenic responses in $d b / d b$ mice.

Interestingly, we observed expression of the Tie-1 receptor, for which a specific ligand has still to be identified, to be increased during normal repair. Upregulation of Tie-1 expression has also been reported to be involved in angiogenesis in metastatic melanomas, brain tumors, and mammary carcinomas (Hatva et al, 1995; Kaipainen et al, 1994; Salven et al, 1996). In vivo data obtained from gene disruption experiments clearly indicate a role for Tie-1 in maintenance of structural integrity of vascular endothelial cells resulting in prevention of edema and hemorrhage during embryonic development (Puri et al, 1995; Sato et al, 1995). Thus, up-regulation of Tie-1 expression during repair might also serve comparable functions in newly formed vessels at the wound site. However, the identity of a possible ligand for Tie-1 during wound repair and the role of elevated Tie-1 expression during impaired healing remains unclear at this stage.

Our findings demonstrate an induction of Ang-2 and VEGF during normal wound healing. Moreover, the wound healing defect in $d b / d b$ mice is associated with elevated levels of Ang-2 in the absence of VEGF induction during skin repair, suggesting that a welldefined Ang-2NEGF gradient might be essential for normal wound healing. Furthermore, our data suggest that application of exogenous VEGF might have a beneficial effect on wound healing in $d b / d b$ mice, especially by readjusting the observed Ang-2/NEGF imbalance underlying the severely impaired angiogenic response in these animals.

\section{Materials and Methods}

\section{Animals}

C57BLKS/J-m +/+Lepr $r^{\mathrm{db}}$ mice $(d b / d b)$ obtained from Jackson Laboratories (Bar Harbor, Maine) were chosen because they exhibit characteristics similar to those of human adult-onset diabetes as a result of a single autosomal recessive mutation on chromosome 4. Only the homozygous animals develop diabetes 
(Coleman, 1982). This mutation inhibits signal transduction of the leptin receptor ObR (Chen et al, 1996; Lee et al, 1996). Balb/c mice were used as a model for normal repair. All animals were 10 weeks of age at the start of the experiment.

\section{Wounding and Preparation of Wounds}

Six full-thickness wounds were created on the backs of female $d b / d b$ mice. Balb/c mice were used as a control for normal healing. Mice were anesthetized with a single intraperitoneal injection of ketamine (80 $\mathrm{mg} / \mathrm{kg}$ body weight)/xylazine (10 $\mathrm{mg} / \mathrm{kg}$ body weight). The hair on the back of each mouse was cut, and the back was subsequently wiped with $70 \%$ ethanol. Six full-thickness wounds $(5 \mathrm{~mm}$ in diameter, 3-4 $\mathrm{mm}$ apart) were made on the back of each mouse by excising the skin and the underlying panniculus carnosus. The wounds were allowed to form a scab. Skin biopsy specimens from four animals were obtained 1 , $3,5,7,10$, and 13 days after injury. At each time point, an area which included the scab and the complete epithelial margin was excised from each individual wound. This was done to exclude unaffected skin regions that were located beyond the wound margins and, accordingly, to obtain comparable biopsies from the individual animals. As a control, a similar amount of skin was taken from the backs of four nonwounded mice. In every experiment, the wounds from four animals ( $n=16$ wounds) and the nonwounded back skin from four animals, respectively, were combined, frozen immediately in liquid nitrogen, and stored at $-80^{\circ} \mathrm{C}$ until used for RNA or protein isolation. All animal experiments were carried out according to the guidelines and with the permission from the local government (Regierungspräsidium Darmstadt) of Hessen, Germany.

\section{In Situ Hybridization}

Mice were wounded as described above. Five and 10 days after injury, wounds were excised and fixed in a 4\% paraformaldehyde (PFA)/phosphate-buffered saline (PBS) solution before they were embedded in tissue-freezing medium. Six- $\mu \mathrm{m}$ serial sections were subsequently analyzed for Ang-2 mRNA expression using the HybriProbe in situ hybridization kit (Biognostik, Göttingen, Germany) as described by the manufacturer. The Ang-2-specific oligonucleotide represented bases 599-627 (5' TAC CTA CGG TCC TCA GTG TCT CTG TAC GT 3') of the published sequence (Maisonpierre et al, 1997). A scrambled oligonucleotide (Control HybriProbe) provided by the manufacturer was used as a negative control. The oligonucleotides were labeled by fluorescein-isothiocyanate (FITC) which allows an immunogenic detection of the hybridized probes by an antifluorescein antibody (Roche Biochemicals, Mannheim, Germany).

\section{Immunohistochemistry}

Mice were wounded as described above. Animals were killed at Days 5 and 10 after injury. Complete wounds were isolated from the middle of the back, bisected, and frozen in tissue-freezing medium. Sixmicrometer frozen serial sections were fixed with acetone and treated for 10 minutes at room temperature with $1 \% \mathrm{H}_{2} \mathrm{O}_{2}$ in PBS to inactivate endogenous peroxidases. They were subsequently incubated over night at $4^{\circ} \mathrm{C}$ with polyclonal antisera against murine Tie-2 or murine CD31 (Santa Cruz, Heidelberg, Germany) (1:50 diluted in phosphate-buffered saline, $0.1 \%$ goat serum albumin), respectively. The slides were subsequently stained with the avidin-biotinperoxidase complex system from Santa Cruz (Heidelberg, Germany) using 3-amino-9-ethylcarbazole as a chromogenic substrate. After development, they were rinsed with water, counterstained with hematoxylin (Sigma, Deisenhofen, Germany), and mounted.

\section{Preparation of Tissue Lysates and Western Blot Analysis}

Skin samples were homogenized in lysis buffer $(1 \%$ Triton X-100; 20 mm Tris/HCl, $\mathrm{pH} 8.0 ; 137$ mM NaCl; $10 \%$ glycerol; $5 \mathrm{~mm}$ ethylenediamine tetraacetic acid; $1 \mathrm{~mm}$ phenylmethylsulfonyl fluoride; $1 \%$ aprotinin; 15 $\mu \mathrm{g} / \mathrm{ml}$ leupeptin). The tissue extract was cleared by centrifugation. Fifty micrograms of total protein from these lysates was separated using sodium dodecyl sulfate-gel electrophoresis. After transfer to a PVDF membrane, Ang-1, Ang-2, Tie-1, or CD 31 protein was detected using polyclonal antibodies (Santa Cruz, Heidelberg, Germany). Tie-2-specific proteins were immunoprecipitated using the antibody as described in the paragraph titled "Immunohistochemistry" from $100 \mu \mathrm{g}$ of total protein of lysates isolated from Balb/c and $d b / d b$ mice and subsequently isolated using protein G-Sepharose. Sepharose-beads were washed three times with TNTG buffer $(20 \mathrm{~mm}$ Tris/Cl, $\mathrm{pH} 7.2$; $150 \mathrm{~mm} \mathrm{NaCl} ; 10 \%$ glycerol; $0.1 \%$ Triton $\mathrm{X}-100$ ). Protein G-Sepharose-bound proteins were eluted by a 5-minute incubation at $95^{\circ} \mathrm{C}$ in Laemmli sample buffer and analyzed by Western blotting. After transfer to a PVDF membrane, immunoprecipitated Tie-2 protein was detected with the antibody used for immunoprecipitation. A secondary antibody coupled to horseradish peroxidase and the ECL detection system were used to visualize Ang-1, Ang-2, Tie-1, Tie-2, or CD 31 specific proteins, respectively. Phenylmethylsulfonyl fluoride, aprotinin, and leupeptin were from Sigma (Deisenhofen, Germany), and the ECL detection system was obtained from Amersham (Freiburg, Germany).

\section{RNA Isolation and RNase Protection Analysis}

RNA isolation was performed as described (Chomczynski and Sacchi, 1987). Thirty micrograms of total RNA from wounded or nonwounded skin, or $20 \mu \mathrm{g}$ of RNA from the cell culture experiments, respectively, were used for RNase protection assays. RNase protection assays were carried out as described previously (Werner et al, 1992). Briefly, DNA probes were cloned into the transcription vector pBluescript II KS $(+)$ (Stratagene, Heidelberg, Germany) and linearized. 
An antisense transcript was synthesized in vitro using T3 or T7 RNA polymerase and $\left[\alpha^{-}{ }^{32} \mathrm{P}\right]$ UTP $(800 \mathrm{Ci} /$ $\mathrm{mmol})$. RNA samples were hybridized at $42^{\circ} \mathrm{C}$ overnight with $100,000 \mathrm{cpm}$ of the labeled antisense transcript. Hybrids were digested with RNases $A$ and $\mathrm{T} 1$ for 1 hour at $30^{\circ} \mathrm{C}$. Under these conditions, every single mismatch is recognized by the RNases. Protected fragments were separated on 5\% acrylamide/8M urea gels and analyzed using a PhosphoImager (Fuji, Straubenhardt, Germany). RNases A and T1 were from Roche Biochemicals (Mannheim, Germany).

\section{Probe cDNAs}

The murine cDNA probes for Ang-1 and -2, Tie-1 and -2, CD31, von Willebrand factor (vWF), or VEGFR-3/ Flt-4, respectively, were cloned by reversetranscriptase polymerase chain reaction (RT-PCR). The cloned cDNA fragments correspond to nucleotides 1412-1678 (for Ang-1), nucleotides 1440-1739 (for Ang-2), nucleotides 3090-3361 (for Tie-1), nucleotides 3039-3356 (for Tie-2), nucleotides 1620-1841 (for CD31), nucleotides 9-311 (for vWF), or nucleotides 3839-4081 (for VEGFR-3/Flt-4) of the published sequences (Davis et al, 1996; Finnerty et al, 1993; Iwama et al, 1993; Maisonpierre et al, 1997; Nichols et al, 1994; Sato et al, 1993; Xie and Muller et al, 1993).

\section{Determination of Relative Expression Levels for Ang-1, -2 and Tie-1, -2 at the Wound Site}

Relative expression levels for Ang-1 and Ang-2 mRNA, or Tie-1 and Tie-2 mRNA, respectively, were assessed by RNase protection assay (see above). This technique allows the direct quantification of relative amounts of specific mRNA transcripts. Thirty micrograms of total RNA from normal and wounded skin (Days 1, 3, 5, 7, and 13 after injury) isolated from control and diabetic animals of two independent animal experiments (total of $n=32$ wounds for every experimental time point) were hybridized against an excess of Ang-1-, Ang-2-, Tie-1-, and Tie-2-specific radiolabeled antisense RNA probes. Ang-1/Ang-2, or Tie-1/Tie-2-protected fragments from normal and diabetic wounds, respectively, were separated on the same gels (5\% acrylamide/8M urea) and analyzed using a Phospholmager (Fuji). After a 2-day exposure of the radiolabeled gels to a Phospholmager screen, Ang-1/Ang-2 or Tie-1/Tie-2 signal strengths of the individual wound-healing time points could be directly compared by a readout of the arbitrary PSL (signal intensity per area) counts as determined by the Phospholmager analysis software program. Finally, total PSL counts were corrected, as the antisense RNA probes differed by the amount of incorporated $\left[\alpha{ }^{-32}\right.$ P]UTP as a direct consequence of cDNA template sequences (for Ang-1: $58 \times \mathrm{T}$; for Ang-2: $52 \times \mathrm{T}$; for Tie-1: $60 \times \mathrm{T}$; for Tie-2: $76 \times \mathrm{T}$ ). Please note that the total counts given in Table 1 could only be compared within the individual subtables, but not between the four subtables because each subtable reflects the total PSL counts that had been obtained from individual RNase protection experiments.

\section{Enzyme-Linked Immunosorbent Assay}

Total protein $(50 \mu \mathrm{g})$ from lysates of nonwounded and wounded skin isolated from control $(\mathrm{Ba} / \mathrm{b} / \mathrm{c})$ and $\mathrm{db} / \mathrm{db}$ mice, respectively, were analyzed for the presence of immunoreactive VEGF $_{165}$ protein by enzyme-linked immunosorbent assay (ELISA) using the Quantikine human VEGF kit (R\&D Systems, Wiesbaden, Germany) as described by the manufacturer.

\section{Acknowledgements}

We thank Miss Nicole Kolb for her excellent technical assistance. We gratefully acknowledge Dr. Martin Kock for his help regarding the animal experiments.

\section{References}

Aronson D and Rayfield EJ (1996). Diabetes and obesity. In: Fuster V, Ross R, and Topol EJ, editors. Atherosclerosis and coronary artery disease. Philadelphia: Lipincott-Raven, 327362.

Baumgartner I, Pieczek A, Manor O, Blair R, Kearney M, Walsh K, and Isner JM (1998). Constitutive expression of phVEGF165 after intramuscular gene transfer promotes collateral vessel development in patients with critical limb ischemia. Circulation 97:1114-1123.

Brown LF, Yeo KT, Berse B, Yeo TK, Senger DR, Dvorak HF, and van de Water L (1992) Expression of vascular permeability factor (vascular endothelial growth factor) by epidermal keratinocytes during wound healing. J Exp Med 176:13751379.

Carmeliet P, Ferreira V, Breier G, Pollefeyt S, Kieckens L, Gertsenstein M, Fahrig M, Vandenhoeck A, Harpal K, Eberhardt C, Declerq C, Pawling J, Moons L, Collen D, Risau W, and Nagy A (1996). Abnormal blood vessel development and lethality in embryos lacking a single VEGF allele. Nature 380:435-439.

Chen H, Charlat O, Tartaglia LA, Woolf EA, Weng X, Ellis SJ, Lakey ND, Culpepper J, Moore KJ, Breitbart RE, Duyk GM, Tepper RI, and Morgenstern JP (1996). Evidence that the diabetes gene encodes the leptin receptor: Identification of a mutation in the leptin receptor gene in $\mathrm{db} / \mathrm{db}$ mice. Cell 84:491-495.

Chomczynski P and Sacchi N (1987). Single-step method of RNA isolation by acid guanidinium thiocyanate-phenolchloroform extraction. Anal Biochem 162:156-159.

Clark RAF (1996). Wound repair. Overview and general considerations. In: Clark RAF, editor. The molecular and cellular biology of wound repair. New York and London: Plenum Press, 3-50.

Coleman DL (1982) Diabetes-obesity syndromes in mice. Diabetes 31:1-6.

Davis S, Aldrich TH, Jones PF, Acheson A, Compton DL, Jain V, Ryan TE, Bruno J, Radziejewski C, Maisonpierre PC, and Yancopoulos GD (1996). Isolation of angiopoietin-1, a ligand for the TIE2 receptor, by secretion-trap expression cloning. Cell 87:1161-1169. 
Dumont DJ, Fong GH, Puri MC, Gradwohl G, Alitalo K, and Breitman ML (1995). Vascularization of the mouse embryo: A study of flk-1, tek, tie, and vascular endothelial growth factor expression during development. Dev Dyn 203:80-92.

Dumont DJ, Gradwohl G, Fong GH, Puri MC, Gertsenstein M, Auerbach A, and Breitman ML (1994). Dominant-negative and targeted null mutations in the endothelial receptor tyrosine kinase, tek, reveal a critical role in vasculogenesis of the embryo. Genes Dev 8:1897-1909.

Dumont DJ, Jussila L, Taipale J, Lymboussaki A, Mustonen T, Pajusola K, Breitman K, and Alitalo K (1998). Cardiovascular failure in mouse embryos deficient in VEGF receptor-3. Science 282:946-949.

Ferrara N, Carver-Moore K, Chen H, Dowd M, Lu L, O'Shea KS, Powell-Braxton L, Hillan KJ, and Moore MW (1996). Heterozygous embryonic lethality induced by targeted inactivation of the VEGF gene. Nature 380:439-442.

Finnerty H, Kelleher K, Morris G, Bean K, Merberg D, Kriz R, Morris J, Sookdeo H, Turner K, and Wood C (1993). Molecular cloning of murine FLT and FLT4. Oncogene 8:22932298.

Fong GH, Rossant J, Gertsenstein M, and Breitman ML (1995). Role of the Flt-1 receptor tyrosine kinase in regulating the assembly of vascular endothelium. Nature 376:66-70.

Frank S, Hübner G, Breier G, Longaker MT, Greenhalgh DG, and Werner $S$ (1995). Regulation of vascular endothelial growth factor expression in cultured keratinocytes. Implications for normal and impaired wound healing. $\mathrm{J}$ Biol Chem 270:12607-12613.

Gale NW and Yancopoulos GD (1999). Growth factors acting via endothelial cell-specific receptor tyrosine kinases: VEGFs, angiopoietins, and ephrins in vascular development. Genes Dev 13:1055-1066.

Hatva E, Kaipainen A, Mentula P, Jaaskelainen J, Paetau A, Haltia M, and Alitalo K (1995). Expression of endothelial cell-specific receptor tyrosine kinases and growth factors in human brain tumors. Am J Pathol 146:368-378.

Holash J, Maisonpierre PC, Compton D, Boland P, Alexander CR, Zagzag D, Yancopoulos DG, and Wiegand SJ (1999). Vessel cooption, regression, and growth in tumors mediated by angiopoietins and VEGF. Science 284:1994-1998.

Iwama A, Hamaguchi I, Hashiyama M, Murayama Y, Yasunaga K, and Suda T (1993). Molecular cloning and characterization of mouse TIE and TEK receptor tyrosine kinase genes and their expression in hematopoietic stem cells. Biochem Biophys Res Commun 195:301-309.

Kaipainen A, Vlaykova T, Hatva E, Bohling T, Jekunen A, Pyrhonen S, and Alitalo K (1994). Enhanced expression of the tie receptor tyrosine kinase mesenger RNA in the vascular endothelium of metastatic melanomas. Cancer Res 54:65716577.

Kukk E, Lymboussaki A, Taira S, Kaipainen A, Jeltsch M, Joukov V, and Alitalo K (1996). VEGF-C receptor binding and pattern of expression with VEGFR-3 suggests a role in lymphatic vascular development. Development 122:38293837.

Lee GH, Proenca R, Montez JM, Carroll KM, Darvishzadeh JG, Lee Jl., and Friedman JM (1996). Abnormal splicing of the leptin receptor in diabetic mice. Nature 379:632-635.
Leibel RL, Chung WK, and Streamson CC (1997) The molecular genetics of rodent single gene obesities. J Biol Chem 272:31937-31940.

Lymboussaki A, Partanen TA, Olofsson B, Thomas-Crusells J, Fletcher CDM, de Waal RMW, Kaipainen A, and Alitalo K (1998). Expression of the vascular endothelial growth factor $C$ receptor VEGFR-3 in lymphatic endothelium of the skin and in vascular tumors. Am J Pathol 153:395-403.

Maisonpierre PC, Goldfarb M, Yancopoulos DG, and Gao G (1993). Distinct rat genes with related profiles of expression define a TIE receptor tyrosine kinase family. Oncogene 8:1631-1637.

Maisonpierre PC, Suri C, Jones PF, Bartunkova S, Wiegand SJ, Radziejewski C, Compton D, McClain J, Aldrich TH, Papadopoulos N, Daly TJ, Davis S, Sato TN, and Yancopoulos GD (1997). Angiopoietin-2, a natural antagonist for Tie2 that disrupts in vivo angiogenesis. Science 277:55-60.

Martin P (1997). Wound healing: Aiming for perfect skin regeneration. Science 276:75-81.

Nichols WC, Cooney KA, Mohlke KL, Ballew JD, Yang A, Bruck ME, Reddington M, Novak EK, Swank RT, and Ginsburg D (1994). von Willebrand disease in the RIIIS/J mouse is caused by a defect outside of the von Willebrand factor gene. Blood 83:3225-3231.

Puri MC, Rossant J, Alitalo K, Bernstein A, and Partanen J (1995). The receptor tyrosine kinase TIE is required for integrity and survival of vascular endothelial cells. EMBO $J$ 14:5884-5891.

Risau W (1997). Mechanisms of angiogenesis. Nature 386: 671-674.

Rivard A, Silver M, Chen D, Kearney M, Magner M, Annex B, Peters K, and Isner JM (1999). Rescue of diabetes-related impairment of angiogenesis by intramuscular gene therapy with adeno-VEGF. Am J Pathol 154:355-363.

Salven $\mathrm{P}$, Joensuu $\mathrm{H}$, Heikkila $\mathrm{P}$, Matikainen MT, Wasenius VM, Alanko A, and Alitalo K (1996). Endothelial Tie growth factor receptor provides antigenic marker for assessment of breast cancer angiogenesis. $\mathrm{Br} \mathrm{J}$ Cancer 74:69-72.

Sato TN, Qin Y, Kozak CA, and Andus KL (1993). Tie-1 and tie-2 define another class of putative receptor tyrosine kinase genes expressed in early embryonic vascular system. Proc Natl Acad Sci USA 90:9355-9358.

Sato TN, Tozawa Y, Deutsch U, Wolburg-Buchholz K, Fujiwara $Y$, Gendron-Maguire M, Gridley T, Wolburg H, Risau W, and Qin Y (1995). Distinct roles of the receptor tyrosine kinases Tie-1 and Tie-2 in blood vessel formation. Nature 376:70-74.

Shalaby F, Rossant J, Yamaguchi TP, Gertsenstein M, Wu XF, Breitman ML, and Schuh AC (1995). Failure of blood island formation and vasculogenesis in Flk-1-deficient mice. Nature 376:62-66.

Stratmann A, Risau W, and Plate KH (1998). Cell typespecific expression of angiopoietin-1 and angiopoietin-2 suggests a role in glioblastoma angiogenesis. Am J Pathol 153:1459-1466.

Suri C, Jones PF, Patan S, Bartunkova S, Maisonpierre PC, Davis S, Sato TN, and Yancopoulos GD (1996). Requisite role of angiopoietin-1, a ligand for the TIE2 receptor, during embryonic angiogenesis. Cell 87:1171-1180. 
Suri C, McClain J, Thurston G, McDonald DM, Zhou H, Oldmixon EH, Sato TN, and Yancopoulos GD (1998). Increased vascularization in mice overexpressing angiopoietin-1. Science 282:468-471.

Thurston G, Suri C, Smith K, McClain J, Sato TN, Yancopoulos GD, and McDonald DM (1999). Leakage-resistant blood vessels in mice transgenically overexpressing angiopoietin-1. Science 286:2511-2514.

Towne JB (1989). Management of foot lesions in the diabetic patient. In: Rutherford RB, editor. Vascular surgery. Philadelphia: WB Saunders, 895.

Valenzuela DM, Griffiths JA, Rojas J, Aldrich TH, Jones PF, Zhou H, McClain J, Copeland NG, Gilbert DJ, Jenkins NA, Huang T, Papadopoulos N, Maisonpierre PC, Davis S, and Yancopoulos GD (1999). Angiopoietins 3 and 4: Diverging gene counterparts in mice and humans. Proc Natl Acad Sci USA 96:1904-1909.
Werner S, Peters KG, Longaker MT, Fuller-Pace F, Banda MJ, and Williams LT (1992). Large induction of keratinocyte growth factor expression in the dermis during wound healing. Proc Natl Acad Sci USA 89:6896-6900.

Wong AL, Haroon ZA, Werner S, Dewhirst MW, Greenberg CS, and Peters KG (1997). Tie2 expression and phosphorylation in angiogenic and quiescent adult tissues. Circ Res 81:567-574.

Xie YM and Muller WA (1993). Molecular cloning and adhesion properties of murine platelet/endothelial cell adhesion molecule 1. Proc Natl Acad Sci USA 90:5569-5573.

Xiong M, Elson G, Legarda D, and Leibovich SJ (1998). Production of vascular endothelial growth factor by murine macrophages: Regulation by hypoxia, lactate, and the inducible nitric oxide synthase pathway. Am J Pathol 153:587-598. 